\title{
Rehabilitación Cardíaca Fase I: Progresión según pasos protocolizados en pacientes cardioquirúrgicos en un hospital público de Chile
}

\author{
María José Oliveros ${ }^{1 A}$, Pablo Sepúlveda ${ }^{1,2 A}$, Pamela Serón ${ }^{1-3 A}$, Rocío Fuentes ${ }^{1 A}$ \\ 1 Departamento de Medicina Interna, Universidad de La Frontera, Temuco. \\ 2 Hospital Dr. Hernán Henríquez Aravena, Temuco. \\ 3 Centro de Excelencia CIGES, Universidad de La Frontera, Temuco. \\ A. Kinesiólogo.
}

Antecedentes: La Rehabilitación Cardíaca disminuye las complicaciones de la cirugía cardíaca y es mundialmente reconocida. A pesar de esto, en Chile su desarrollo ha sido lento y el reporte de experiencias bajo.

Objetivo: Describir la progresión de acuerdo los pasos de rehabilitación y días postoperatorios en pacientes de rehabilitación cardíaca fase I en un hospital público de Temuco, Chile.

Metodología: Se analizó una serie de casos de pacientes en rehabilitación cardíaca fase I, participantes de un protocolo basado en hitos motores de seis pasos: movilización en cama (P1); sedente borde cama (P2); marcha de $35 \mathrm{mts}$ (P3); marcha hasta $100 \mathrm{mts}$ (P4); marcha hasta 200 metros más subir/bajar un piso de escaleras (P5); marcha de 300 metros más subir/bajar dos pisos de escaleras (P6).

Resultados: Se incluyeron 243 pacientes (1.033 sesiones) con una media de 65.9 años (DS 9.5), mediana de hospitalización 6 días. Del total de atenciones realizadas el día 1, en 53\% de ellas se alcanzó el P1; en el día 2, en un $51 \%$ se alcanzó el P2 y en un $42 \%$ un paso $\geq P 3$; en el día 3 , en $44.78 \%$ se alcanzó paso $\geq P 4$; en el día 4 , en $37.75 \%$ se alcanzó $\geq P 4$. En los días 5-7, aproximadamente $50 \%$ logró $\geq \mathrm{P} 5$.

Conclusión: La progresión de los pasos es proporcional al día postcirugía y es más rápida en hombres. $\mathrm{Al}$ momento del alta, $75 \%$ de los pacientes alcanza una independencia funcional que les permite caminar dos cuadras y subir/bajar un piso de escaleras.

Correspondencia:

Rocío Fuentes Aspe.

rocio.fuentes@ufrontera.cl 


\section{Phase I Cardiac Rehabilitation: Progression through protocolized steps in cardiosurgical in-patients in a public hospital in Chile}

Background: Despite advances in cardiovascular disease management and the development of minimally invasive approaches, open cardiac surgery is still a preferred intervention in complicated cases. Phase I Cardiac Rehabilitation decreases complications associated with this intervention.

Globally, cardiac rehabilitation is widely recommended. However, in Chile the implementation of this intervention has been slow and there are scarce reports of its result.

Objective: To describe the progression according to steps and post-surgery days in cardiac rehabilitation phase I patients in the public hospital in Temuco, Chile.

Methodology: We analyzed a series of cases of patients in cardiac rehabilitation phase I, participants of a protocol based on six-step motor levels: (S1) onbed mobilization, (S2) sitting on the edge of the bed, (S3) Bipedal exercise and 35 meter walking, (S4) 100 meter walking, (P5) 200 meter walking plus going up one floor and down stairs, and (S6) 300 meter walking plus going two floors up and down stairs.

Results: 243 patients were included, and 1,033 sessions were performed) Mean age was 65.9yearsold (SD 9.5) and hospital stay run for a median of 6 days. From all sessions, on day one, $53 \%$ of patients reached $\mathrm{S} 1$. On day 2, 51\% reached S2 and $36 \%$ walked (S3). On day 3, 44.78\% reached $\geq \mathrm{S} 4$. On day $4,37.75 \%$ reached $\geq \mathrm{S} 4$. On days $5-7$, approximately $50 \%$ achieved $\geq$ S5 .

Conclusion: the step progression is proportional to number of days after surgery, faster in men than compared to women. At discharge, $75 \%$ of patients show functional independency that allows them to walk two blocks and go one floor up and down stairs.

Keywords: Cardiac Rehabilitation, Cardiac Surgery, protocols. 


\section{Introducción:}

A pesar de los avances en el tratamiento de la enfermedad cardiovascular y el desarrollo del enfoque mínimamente invasivo, la cirugía cardíaca continúa siendo la intervención de elección en casos donde otras alternativas no pueden ser utilizadas. La Asociación Americana del Corazón ha reportado un aumento del $28 \%$ en el total de operaciones y procedimientos cardiovasculares entre los años 2000 y 2010. Aunque la tendencia de los últimos años pareciera ir en disminución, continúa siendo una de las condiciones que genera mayor número de procedimientos quirúrgicos ${ }^{1}$. En países de altos niveles de ingresos como Norteamérica, Australia y Europa esta cifra alcanza a 860 por un millón de personas y en países de menos ingresos, como en Sudamérica alcanza un promedio de 60 cirugías por un millón ${ }^{2}$. La cirugía de revascularización miocárdica es efectiva en el tratamiento de la enfermedad coronaria, y en pacientes de mayor riesgo ha mostrado mayor efectividad que la intervención coronaria percutánea ${ }^{3,4}$. De igual forma la cirugía de recambio valvular es el tratamiento predominante para la enfermedad cardíaca valvular moderada y severa ${ }^{5}$. A pesar de los resultados globales favorables, la cirugía es una intervención invasiva que provoca alteraciones fisiológicas importantes como inestabilidad hemodinámica, alteraciones en el sistema respiratorio, además de la reducción de la capacidad física asociada a la situación de salud previa y posteriormente a la situación de hospitalización ${ }^{6}$. La Rehabilitación Cardiaca (RC) fase I busca disminuir las complicaciones asociadas a la intervención y la hospitalización, optimizando la función ventilatoria, favoreciendo la movilización precoz y por ende lograr una mayor capacidad funcional al momento del alta. Si bien en la actualidad la movilización precoz en las unidades de cuidados intensivos es una práctica cada vez más habitual, esta tendencia tardó un largo tiempo en convertirse en rutina ${ }^{7}$. En el año 1936 junto con la primera descripción anatomopatológica de necrosis miocárdica, aún se discutía sobre el reposo de seis a ocho semanas en cama y alrededor de seis meses en un sillón en sujetos post infarto agudo al miocardio ${ }^{8}$. Treinta años más tarde, en la década del sesenta, un período de descanso de tres semanas seguía siendo común después de un infarto ${ }^{9}$. En el año 1964, la Organización Mundial de la Salud, considera el ejercicio físico en pacientes con enfermedad coronaria, dentro de la $\mathrm{RC}$ como una intervención integral. A partir de entonces se comienzan a desarrollar los primeros programas de $\mathrm{RC}^{9}$, intervención que hoy es mundialmente conocida y recomendada ${ }^{10}$. A pesar de esto su desarrollo ha sido lento. En América Latina, al año 2013 tan solo el $50 \%$ de los centros que reportó el servicio de $\mathrm{RC}$ realizaban fase $\mathrm{I}^{11}$, situación que no difiere mucho en Chile dónde esta cifra alcanza sólo un $42 \% 12$. Sumado a esto, el reporte de experiencias en fase I, hasta ahora van orientadas mayoritariamente a la obtención de un hito motor específico basado en el día post-cirugía. Sin embargo, debido a las distintas características personales, clínicas, de la intervención o del entorno, las respuestas al esfuerzo post cirugía no son iguales para todos, por lo que si la rehabilitación se basa sólo en el día post-cirugía e hito motor correspondiente a realizar, en muchos casos se podría sobre o subestimar la intensidad de la rehabilitación.

En este contexto, el presente estudio tiene como objetivo describir los resultados de la aplicación de un protocolo de RC fase I basado en el logro de hitos motores tras una evaluación segura en sujetos sometidos a cirugía cardíaca en el Hospital Dr. Hernán Henríquez Aravena de Temuco, relacionando los pasos de rehabilitación alcanzados, con los días post cirugía.

\section{Método}

Se analizó la evolución intrahospitalaria de pacientes sometidos a cirugía cardíaca (revascularización miocárdica o recambio valvular) en el Hospital Dr. Hernán Henríquez Aravena de Temuco en Chile, entre marzo del año 2016 y octubre del año 2017. Desde el postoperatorio inmediato, los pacientes con indicación médica de kinesioterapia fueron incluidos en un programa de RC fase I, basado en un protocolo de movilización temprana, según el "Semáforo de atención segura en RC fase 1" elaborado como parte de este protocolo (Figuras 1a-1b ). La elaboración de este semáforo consideró reportes previos de atención segura en unidades de cuidados intensivos ${ }^{13,14}$, adaptados específicamente para una unidad de cuidados intensivos cardiovascular. Este proceso fue conducido por las autoras de este trabajo e incluyó la participación tanto de médicos como enfermeros de la Unidad de Paciente Crítico Cardiovascular del Hospital Dr. Hernán Henríquez Aravena. El "Semáforo de atención segura en RC fase 1" se construyó para valorar la condición diaria del paciente y se constituyó en tres dominios: consideraciones cardiovasculares, respiratorias y otros. A su vez, cada dominio, incluyó ítems con consideraciones más específicas relacionadas a parámetros de seguridad, diagnóstico y pronóstico, estabilidad, uso de fármacos y elementos de apoyo. Finalmente, cada ítem específico fue categorizado en colores: verde (bajo riesgo de complicación), 


\begin{tabular}{|c|c|c|c|}
\hline \multicolumn{4}{|l|}{ Figura 1.} \\
\hline & $\begin{array}{l}\text { KNT en } \\
\text { cama }\end{array}$ & $\begin{array}{l}\text { Fuera de } \\
\text { cama }\end{array}$ & Observaciones \\
\hline \multicolumn{4}{|c|}{ CONSIDERACIONES CARDIOVASCULARES } \\
\hline $\begin{array}{l}\text { Enfermedad TCl } \\
\text { Disección aórtica tipo A }\end{array}$ & & & Evaluar beneficio de KTR \\
\hline $\begin{array}{l}\text { Angina inestable / } \\
\text { Disección aórtica tipo B }\end{array}$ & & & Consenso equipo. \\
\hline $\begin{array}{l}\text { Arritmias no controladas } \\
\text { (auriculares ventriculares) }\end{array}$ & & & $\begin{array}{l}\text { Evaluar estabilidad hemodinámica. } \\
\text { Taquicardia > } 120 \text { por minuto }\end{array}$ \\
\hline $\begin{array}{l}\text { Balón contrapulsación } \\
\text { intraaortico }\end{array}$ & & & $\begin{array}{l}\text { Posibilidad KTR. } \\
\text { Contraindicado flexión cadera sitio }\end{array}$ \\
\hline $\begin{array}{l}\text { Sospecha nueva isquemia } \\
\text { cardiaca / cambios ECG }\end{array}$ & & & Consenso equipo. \\
\hline Embolismo reciente & & & $\begin{array}{l}\text { KNT respiratoria, evitar KNT motora } \\
\text { Esperar } 24 \text { horas del inicio tto. }\end{array}$ \\
\hline $\begin{array}{l}\text { Bradicardia cl } \\
\text { requerimiento } \\
\text { farmacológico o en espera } \\
\text { de MP. }\end{array}$ & & & $\begin{array}{l}\text { C/ MP externo puede pasar a sillón y } \\
\text { movilizarse con precaución. }\end{array}$ \\
\hline $\begin{array}{l}\text { Dependiente } \\
\text { marcapaso externo }\end{array}$ & & & Seguridad de la conexión. \\
\hline $\begin{array}{l}\text { IC descompensada } \\
\% \mathrm{FE} \leq 40 \%\end{array}$ & & & $\begin{array}{l}\text { Esfuerzo liviano Borg } 10 \text { - 11, } \\
\text { interválico. }\end{array}$ \\
\hline Hemoglobina & & & $\begin{array}{l}\text { Bajo } 8 \mathrm{mg} / \mathrm{DL} \text { evitar levantar o } \\
\text { progresivo }\end{array}$ \\
\hline \multicolumn{4}{|c|}{$\begin{array}{l}\text { PRESIÓN ARTERIAL } \\
\text { - PAS: } 80-139 \mathrm{~mm} \mathrm{Hg} \\
\text { - PAD: } 55-89 \mathrm{~mm} \mathrm{Hg} \\
\text { - PAM: } 60-90 \mathrm{~mm} \mathrm{Hg}\end{array}$} \\
\hline \multicolumn{4}{|l|}{$\begin{array}{l}\text { Fuera rango normalidad } \\
\text { con síntomas }\end{array}$} \\
\hline \multicolumn{4}{|l|}{$\begin{array}{l}\text { Fuera rango normalidad } \mathrm{cl} \\
\text { alto nivel de soporte } \mathrm{VA}\end{array}$} \\
\hline \multicolumn{4}{|l|}{$\begin{array}{l}\text { En rango normalidad } \mathrm{cl} \\
\text { moderado nivel de soporte }\end{array}$} \\
\hline $\begin{array}{l}\text { En el límite normalidad sin } \\
\text { soporte o } \\
\text { bajos niveles de soporte }\end{array}$ & & & \\
\hline
\end{tabular}

rojo (alto riesgo de complicación) y amarillo (evaluar si corresponde a bajo o alto riesgo de complicación) para la atención kinésica en cama o fuera de la cama, lo que permite orientar la posterior intervención.

Las intervenciones consideradas en este programa de $\mathrm{RC}$ fase I incluyeron kinesioterapia respiratoria para prevenir o tratar complicaciones según cada caso y una activación cardiovascular progresiva a través de kinesioterapia motora y el logro de hitos motores o pasos in- dependientes del día de evolución postquirúrgica. Estos hitos fueron organizados de forma progresiva en 6 pasos $(\mathrm{P})$, basados en la escasa experiencia previa publicada ${ }^{6}$ y literatura disponible en textos clásicos ${ }^{15}$ adaptado a la realidad local. Los pasos son: P1=movilización en cama, P2 = sedestación al borde de la cama, P3= bipedestación y deambulación hasta 35 metros (dentro de la unidad), P4= deambulación hasta 100 metros, P5= deambulación entre 100 y 200 m, más subir y bajar un piso por las escaleras, 


\section{TABLA 1: PASOS INCLUIDOS EN EL PROTOCOLO DE MOVILIZACIÓN TEMPRANA COMO PARTE DE LA RC FASE 1}

ETAPA *

DOSIFICACIÓN/ GASTO EN MET: Sesiones aproximadas 30 minutos. 2 veces por día.

FC de seguridad calculada 30\% FC de reserva. Intensidad Percibida en Escala de Borg modificada 2-3

Gasto calórico aproximado: 2 METs.

\begin{tabular}{|c|c|c|}
\hline PASO 1 & $\begin{array}{l}\text { Paciente en supino. } \\
\text { Invadido } \\
\text { (VM o no) } \\
\text { (drenajes) }\end{array}$ & $\begin{array}{l}\text {-Angulación, si es posible sentar. } \\
\text {-Ejercicios de respiración diafragmática + patrones ventilatorios. } \\
\text {-Inicios incentivadores ventilatorios. } \\
\text {-Técnicas mantener vía aérea permeable. } \\
\text {-Ejercicios pasivos, activos y activos-asistidos de las extremidades. } \\
\text {-Favorecer evacuación drenajes. }\end{array}$ \\
\hline PASO 2 & $\begin{array}{l}\text { Paciente con posibilidad } \\
\text { de sentarse }\end{array}$ & $\begin{array}{l}\text { •Ejercicios posición sedente (borde cama o sillón). } \\
\text {-Ejercicios de respiración diafragmática + patrón ventilatorio. } \\
\text {-Ejercicios con incentivos ventilatorio. } \\
\text {-Ejercicios activos de las extremidades. } \\
\text {-Ejercicios con cicloergómetro portátil. } \\
\text {-Bípedo, marcha en el lugar. } \\
\text { - Favorecer evacuación drenajes }\end{array}$ \\
\hline
\end{tabular}

DOSIFICACIÓN/ GASTO EN MET: Sesiones aproximadas 15-20 minutos. 2 veces por día.

FC de seguridad calculada 30\% FC de reserva. Intensidad Percibida en Escala de Borg modificada 2-3

Gasto calórico aproximado: 3 - 3.5 METs.

\begin{tabular}{|c|c|c|}
\hline PASO 3 & $\begin{array}{l}\text { Paciente con posibilidad } \\
\text { de ponerse de pie }\end{array}$ & $\begin{array}{l}\text {-Ejercicios sentado y de pie } \\
\text {-Ejercicios con incentivos ventilatorio } \\
\text {-Ejercicios activos y movilización en máximo rango de las extremidades. } \\
\text {-Ejercicios con cicloergómetro portátil } \\
\text {-Ejercicios con banda elástica (tensión leve) } \\
\text {-Deambulación por unidad hasta } 35 \mathrm{~m} \text {. }\end{array}$ \\
\hline PASO 4 & $\begin{array}{l}\text { Paciente de pie y } \\
\text { deambulando con apoyo }\end{array}$ & $\begin{array}{l}\text {-Ejercicios de pie } \\
\text { •Ejercicios con incentivos ventilatorio } \\
\text {-Ejercicios activos y movilización en máximo rango de las extremidades. } \\
\text {-Ejercicios con cicloergómetro portátil } \\
\text {-Ejercicios con banda elástica (tensión leve) } \\
\text {-Deambulación por pasillo de } 60 \text { a } 100 \mathrm{~m} .\end{array}$ \\
\hline PASO 5 & $\begin{array}{l}\text { Paciente de pie y } \\
\text { deambulando sin apoyo }\end{array}$ & $\begin{array}{l}\text {-Ejercicios de pie } \\
\text {-Ejercicios con incentivos ventilatorio } \\
\text {-Ejercicios activos y elongaciones de las extremidades } \\
\text {-Ejercicios con cicloergómetro portátil } \\
\text {-Ejercicios con banda elástica (tensión leve) } \\
\text { - Deambulación por pasillo de } 100 \text { a } 200 \mathrm{~m} \text {. } \\
\text {-Subir y bajar escaleras } 1 \text { piso escalera }\end{array}$ \\
\hline PASO 6 & $\begin{array}{l}\text { Paciente de pie y } \\
\text { deambulando sin apoyo }\end{array}$ & $\begin{array}{l}\text { - Ejercicios de pie } \\
\text {-Ejercicios con incentivos ventilatorio } \\
\text {-Ejercicios activos y elongaciones de las extremidades } \\
\text {-Ejercicios con cicloergómetro portátil } \\
\text {-Ejercicios con banda elástica (tensión leve) } \\
\text { - Deambulación por pasillo de } 300 \mathrm{~m} \text {. } \\
\text {-Subir y bajar escaleras } 1-2 \text { piso escalera }\end{array}$ \\
\hline
\end{tabular}


y P6= deambulación entre 200 y 300 m, más subir y bajar dos pisos por las escaleras. Más detalles del protocolo en Tabla 1. Las sesiones fueron de intensidad liviana según la tabla de intensidades propuesta por el Colegio Americano de Medicina del Deporte ${ }^{16}$, con actividades e hitos motores hasta $3.5 \mathrm{METs}^{17}$, monitorizándose que la percepción de esfuerzo a través de la escala de Borg modificada fuese entre 2-3, y resguardando un porcentaje de carga cardíaca máximo de $30 \%$ de la frecuencia cardíaca de reserva, considerado como frecuencia cardíaca de seguridad.

La recolección de datos se realizó diariamente en una planilla diseñada para este propósito que contenía información sobre características de los sujetos (edad, sexo),

\begin{tabular}{lc}
\hline TABLA 2: Características de los sujetos & \\
\hline VARIABLE & RESULTADO \\
Edad (años) & $63.7 \pm 10.7$ \\
Sexo (masculino) & $72.81 \%$ \\
\hline TIPO CIRUGíA & \\
Revascularización & $66 \%$ \\
Valvular o mixta & $34 \%$ \\
\hline DíAS DE HOSPITALIZACIÓN (MEDIANA) & 7 \\
Mínimo & 3 \\
Máximo & 55 \\
\hline
\end{tabular}

descripción de la intervención y días de hospitalización (Tabla 2). Las variables principales: días post-cirugía y el paso de rehabilitación alcanzado se registró al finalizar

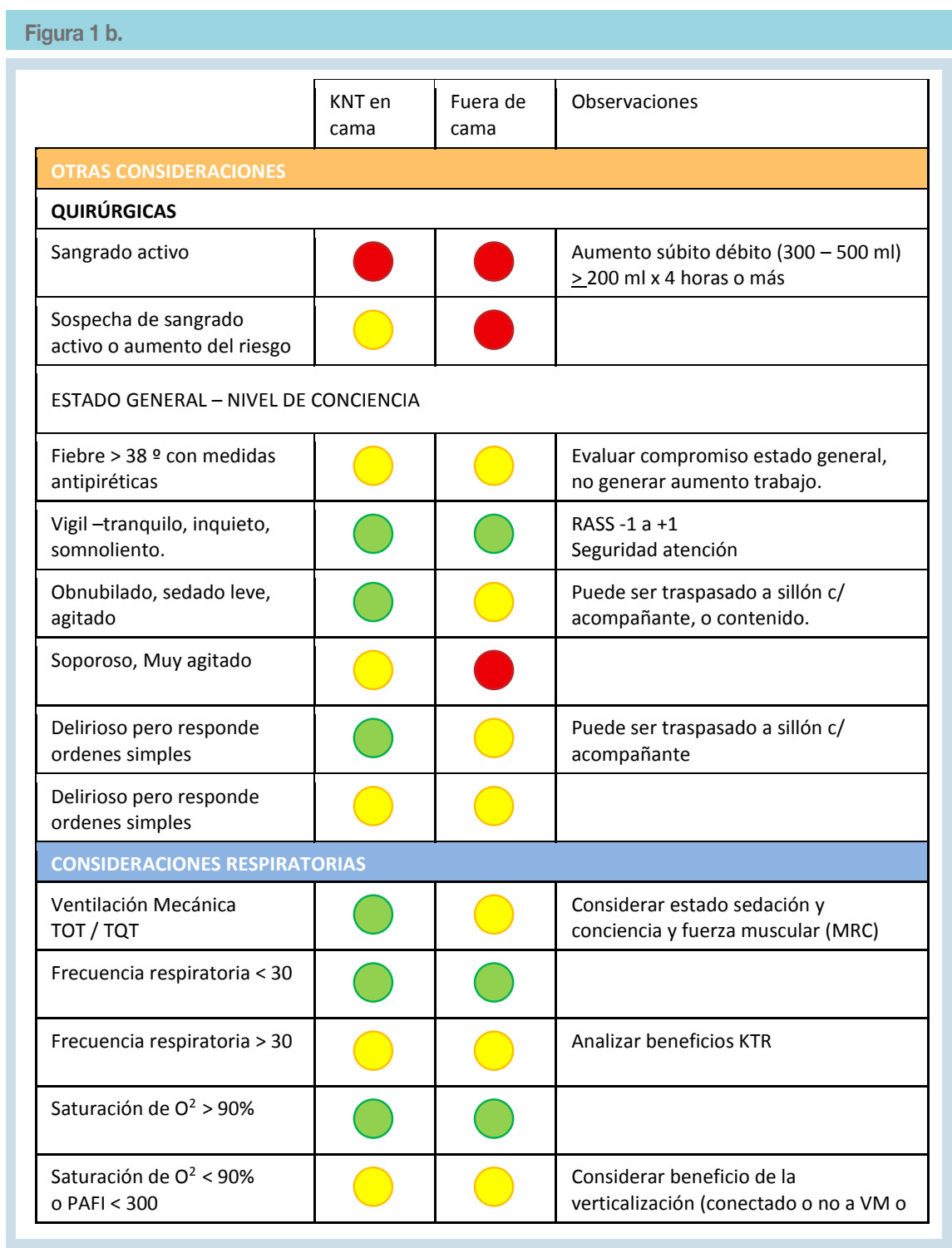




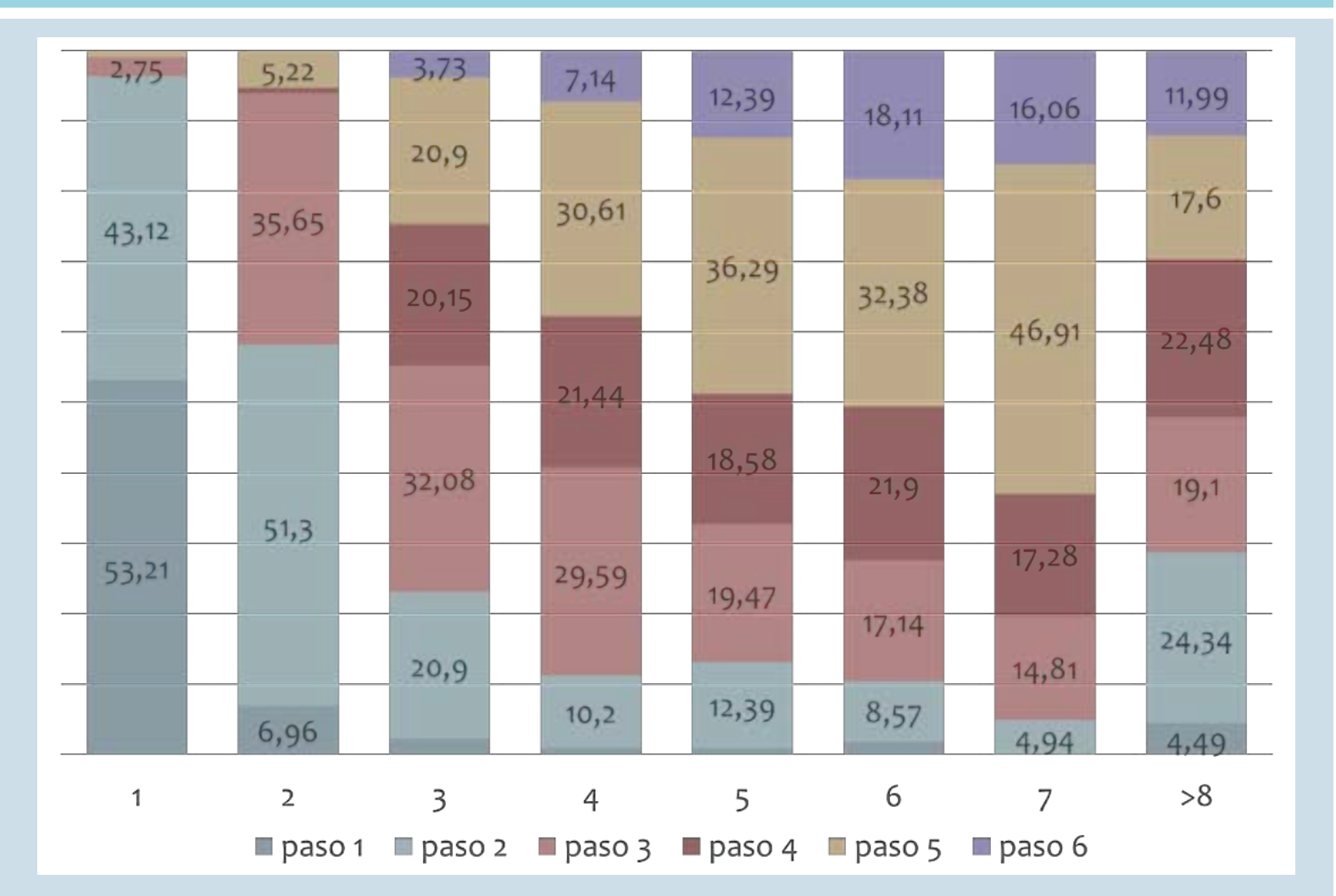

cada atención. Para los días post cirugía se consideró el día de la cirugía según calendario como día cero y para definir el paso de rehabilitación alcanzado se utilizó el protocolo antes descrito.

Se utilizaron medidas de tendencia central y proporciones para describir las características de la muestra de estudio. Se realizó análisis estratificado para explorar las variables demográficas y clínicas relacionadas a la evolución de los pacientes. Se excluyeron del análisis los sujetos con datos de interés faltantes o menos de dos atenciones de RC durante la hospitalización.

\section{Resultados:}

En el período de marzo de 2016 a octubre de 2017 se realizaron 1033 atenciones kinésicas correspondientes a 230 pacientes. $73 \%$ de los pacientes eran hombres y el promedio de edad fue de $63.7+10.7$ años, siendo las mujeres 3,9 años mayores que los hombres $(\mathrm{p}<0,05)$. El 66\% de los pacientes fue sometido a una cirugía de revascularización miocárdica significativamente más frecuente en hombres (71\% en hombres vs $53 \%$ en mujeres), y el otro $34 \%$ fue sometido a una cirugía valvular o mixta (cirugía valvular más revascularización). La mediana de hospitalización fue de 7 días, siendo 6 días en las cirugías de recambio valvular versus 7 en las cirugías de revascularización miocárdica $(\mathrm{p}<0,05)$; no existió diferencia en los días de hospitalización según sexo.

La Figura 2 muestra la evolución de los pacientes por día y pasos alcanzados. Del total de atenciones realizadas el día 1, en el 53\% de ellas se alcanzó el P1 -movilizaciones activas asistidas en cama $\neg-$ y en un $43 \%$ se logró el P2 -posición sedente al borde de la cama $\neg-$ ( $46 \%$ en hombres v/s $33 \%$ en mujeres). En las atenciones realizadas en el día 2 , en un $51 \%$ se alcanzó el P2, mientras que en un $42 \%$ se alcanzó al menos el P3. En el día 3 , en un $32,1 \%$ de las atenciones realizadas se logró el P3 -caminar en la unidad hasta 35 metros $\neg-\mathrm{y}$ en un $44.78 \%$ se alcanzó al menos el P4. En el día 4, un $21.4 \%$ alcanzó el P4 -deambulación hasta $100 \mathrm{~m} \neg-\mathrm{y}$ un $37.8 \%$ un paso superior. Los días 5-7, aproximadamente, el 50\% logró al menos el P5. En las atenciones realizadas en sujetos que estuvieron hospitalizados 8 días o más, el $84 \%$ se distribuyó entre el paso 2 al 5, 


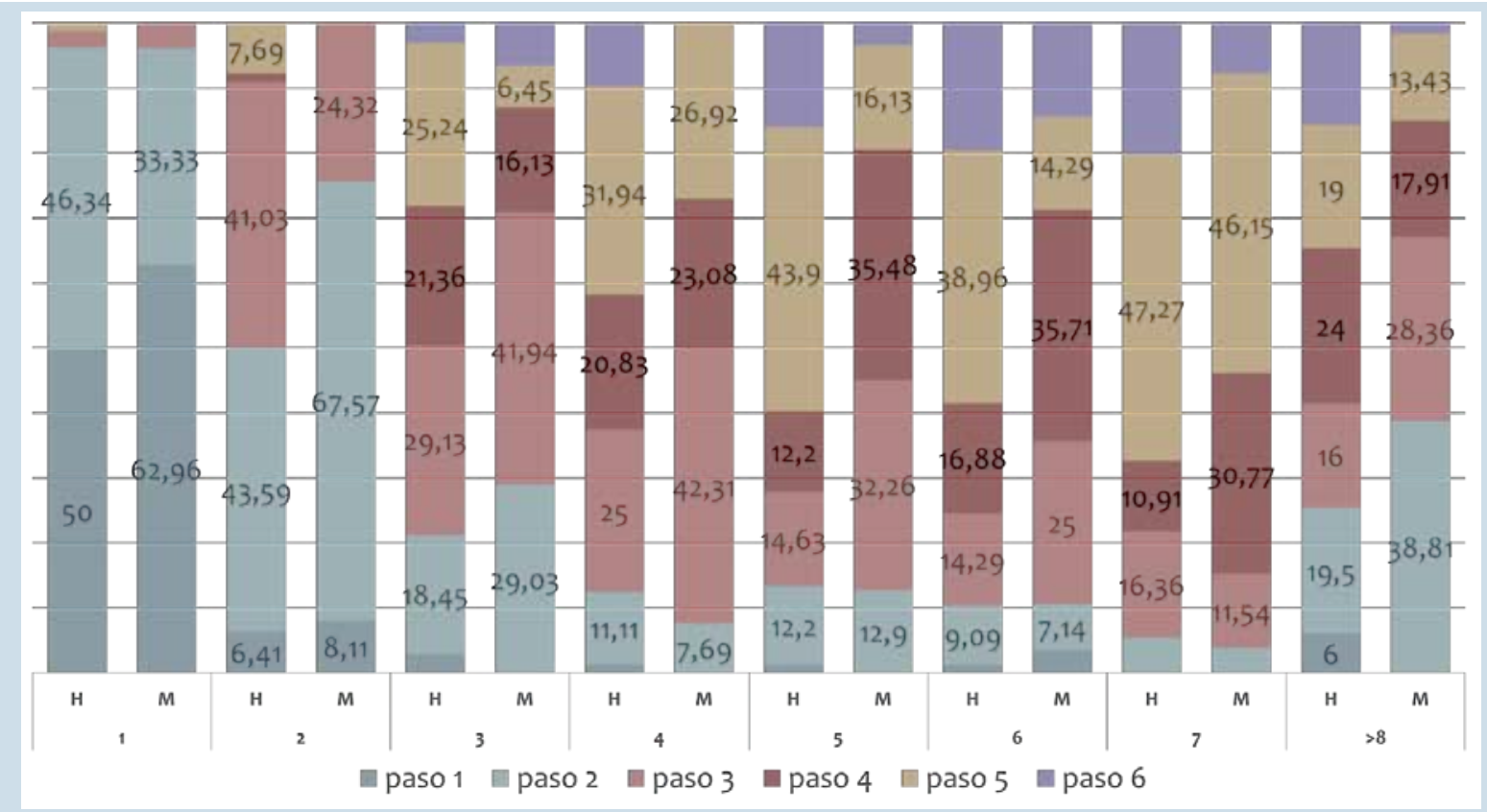

y el $16 \%$ restante en los pasos más extremos 1 o 6. En el $75 \%$ de las atenciones realizadas el día del alta, los sujetos alcanzaron al menos el P5.

En la progresión de los pasos de rehabilitación según sexo, se observó una tendencia positiva a favor del sexo masculino, alcanzando pasos mayores que las mujeres en todos los días post cirugía, como se muestra en detalle en la Figura 3.

Según el tipo de cirugía, se observó un comportamiento similar en el logro de pasos de rehabilitación en los días 1, 2 y 7, observándose una tendencia a alcanzar pasos mayores en sujetos sometidos a cirugía de revascularización versus cirugías valvulares en las atenciones realizadas entre los días 3-6. En las atenciones realizadas en sujetos hospitalizados $\geq 8$ días, aproximadamente, el $50 \%$ de las atenciones se realizó con deambulación fuera de la unidad, independientemente del tipo de cirugía (ver Figura 4).

En cuanto a la presencia de elementos accesorios, se observó que, a medida que se avanza en el postoperatorio, el paciente se encuentra menos invadido y alcanza pasos mayores. Específicamente, se observó que en $11 \%$ de las sesiones que se realizaron en sujetos considerados como parcialmente invadidos (catéter venoso y arterial, drenajes, sonda Foley, sonda nasogástrica, elementos de oxigenoterapia) se alcanzó al menos el P4.

\section{Discusión:}

Nuestros resultados muestran que, si bien la progresión de los pasos de rehabilitación se relaciona de manera directamente proporcional al día post cirugía, no debiera ser considerado un planteamiento rígido y estricto de actuación, ya que el ritmo en el logro de los pasos también tuvo relación con el tipo de intervención y sexo del sujeto.

Respecto a la progresión de la rehabilitación según sexo, se demostró una tendencia positiva a favor del sexo masculino, en todos los días post cirugía. Nuestros hallazgos son consistentes con otros estudios. La observación más aceptada es que las mujeres que necesitan una intervención de cirugía cardíaca tienen mayor riesgo que los hombres, relacionada con su mayor edad, enfermedad más avanzada, más comorbilidades y área de superficie corporal más pequeña ${ }^{18,19}$.

Según el tipo de cirugía observamos un comportamiento similar en el logro de pasos de rehabilitación en los primeros y últimos días de hospitalización, pero con una tendencia a alcanzar pasos mayores en sujetos sometidos a cirugía de revascularización versus cirugías valvulares en las atenciones realizadas entre el tercer y sexto días. Específicamente, en el caso de cirugías valvulares existen importantes diferencias entre hombres y mujeres en 


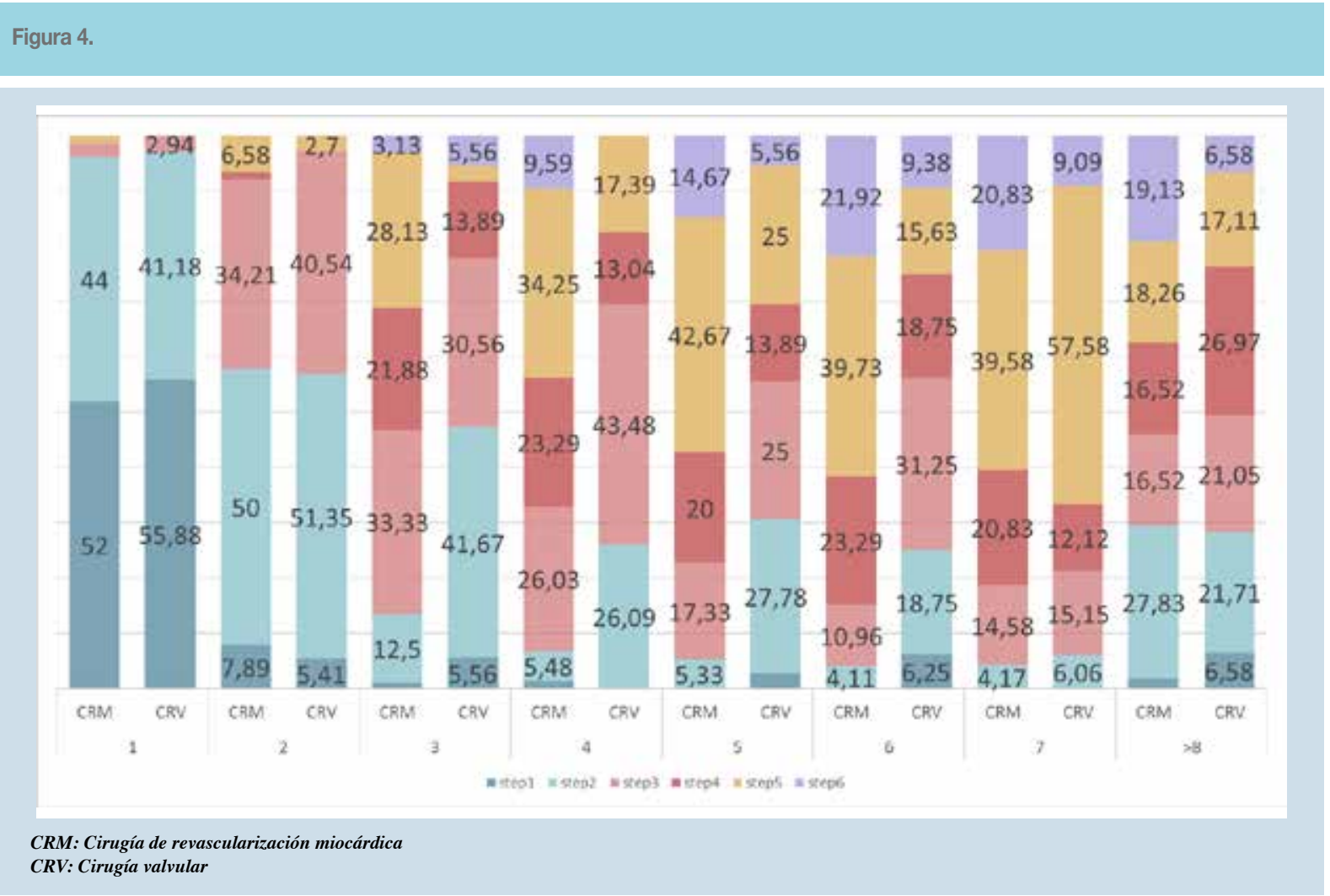

cuanto a las características del paciente o de la intervención, siendo las pacientes de sexo femenino mayores, con una enfermedad más grave en el momento de la cirugía y, mayoritariamente, sometidas a recambios valvulares y en menor medida a reparación de la válvula ${ }^{18}$.

En cuanto a la relación entre el paso alcanzado y la presencia de elementos accesorios, se observó que si bien hay una tendencia a estar menos invadido según aumenta el día post operatorio, la presencia de elementos accesorios no limita en gran medida el logro de pasos de rehabilitación.

Otras características que pudieran asociarse con el logro de los pasos de rehabilitación, pero que no fueron consideradas en el presente trabajo, son relacionadas al sujeto (como la edad, comorbilidades, aptitud física, motivación o respuesta a la intervención), a la intervención (como complejidad, tiempo de cirugía y complicaciones), y al entorno de salud y terapeuta (como experiencia del terapeuta, coordinación del equipo de salud, características de la unidad), las cuales debieran ser consideradas en futuros estudios.

Otros estudios han reportado el progreso o movilizaciones realizadas durante la hospitalización en la fase I de RC. En el estudio de Winkelmann en 2015, se observó que en cada día post cirugía los pacientes evolucionaban logrando pasos mayores, donde la bipedestación y marcha de 35 metros fue lo más utilizado ${ }^{6}$. En el estudio de Overend 2010 realizado en hospitales de Canadá, se reportó un alto porcentaje de sedestación al borde de cama en el primer día postoperatorio, y deambulación incluso fuera de la unidad desde el segundo día postoperatorio ${ }^{20}$. Estos hallazgos difieren de lo encontrado en nuestro estudio lo que podría ser explicado, en parte, por el contexto de la atención, como el tipo de insumos utilizados para el drenaje mediastínico, los cuales no permiten la movilización más allá de la unidad del paciente, siendo el segundo día el más frecuente para el retiro de estos drenajes.

Esta propuesta de rehabilitación cardíaca fase I, basado en un protocolo de movilización temprana utilizando el "Semáforo de atención segura en RC" como parámetro de actuación para el logro de hitos motores, permite alcanzar el máximo hito motor posible en una atención favoreciendo la movilización precoz, lo que contribuye a la disminución de las complicaciones asociadas a la atención kinésica, considerado las características individuales en de cada paciente. Este abordaje basado en una evaluación diaria y que de ella se desprenda una atención segura para lograr hitos y no exclusivamente lo esperado por día de 
evolución postcirugía, como se hace tradicionalmente, permite personalizar la atención diaria de los pacientes sin subestimar ni sobreestimar la rehabilitación.

Las limitaciones observadas en este reporte se basan en el carácter descriptivo del diseño, que no permite establecer asociaciones causales. Sin embargo, el tamaño de muestra, el marco temporal incluido y la pertinencia de los análisis, permiten tener una descripción adecuada de las experiencias de RC Fase I en un Hospital público de Chile.

Finalmente, se puede concluir que la progresión de los hitos motores durante la RC Fase I se observa proporcional al día de cirugía, con una tendencia a que los hombres logren pasos mayores. En general, los sujetos sometidos a RC Fase I luego de una cirugía cardíaca, al momento del alta logran una independencia funcional que les permite caminar dos cuadras, bajar y subir un piso de escaleras.

\section{Agradecimientos}

Los autores agradecen la colaboración de todo el equipo de salud de la Unidad de Paciente Crítico Cardiovascular del Hospital Dr. Hernán Henríquez Aravena; y en especial, a los internos de la carrera de Kinesiología de la Universidad de La Frontera que formaron parte de este equipo durante su práctica profesional.

\section{Referencias}

1. GO AS, MOZAFFARIAN D, ROGER VL, BENJAMIN EJ, BERRY JD, BORDEN WB, et al. AHA Statistical Update Heart Disease and Stroke Statistics - 2013 Update A Report From the American Heart Association WRITING GROUP MEMBERS. Circulation. 2013;January(2).

2. REICHERT HA, RATH TE. Cardiac Surgery in Developing Countries. J Extra Corpor Technol [Internet]. 2017 [cited 2018 May 14];49(2):98-106. Available from: http://www.ncbi.nlm. nih.gov/pubmed/28638158.

3. BUNTAINE AJ, SHAH B, LORIN JD, SEDLIS SP. Revascularization Strategies in Patients with Diabetes Mellitus and Acute
Coronary Syndrome. Curr Cardiol Rep [Internet]. 2016; Available from: http://dx.doi.org/10.1007/s11886-016-0756-3

4. BANGALORE S, GUO Y, SAMADASHVILI Z, BLECKER S, XU J, HANNAN EL. Everolimus Eluting Stents Versus Coronary Artery Bypass Graft Surgery for Patients With Diabetes Mellitus and Multivessel Disease. 2016;

5. RIBEIRO GS, TARTOF SY, OLIVEIRA DWS, GUEDES ACS, REIS MG, RILEY LW, et al. Surgery for Valvular Heart Disease : A Population-Based Study in a Brazilian Urban Center. 2012;7(5). 
6. WINKELMANN ER, DALLAZEN F, BRONZATTI ABS, LORENZONI JCW, WINDMÖLLER P. Analysis of a STEPs adapted protocol in Cardiac Rehabilitation in Phase Hospital. Rev Bras Cir Cardiovasc [Internet]. 2014;40-8. Available from: http://www.gnresearch.org/doi/10.5935/1678-9741.20140048.

7. SANTOS PMR, RICCI NA, SUSTER ÉAB, PAISANI DM, CHIAVEGATO LD. Effects of early mobilisation in patients after cardiac surgery: a systematic review. Physiotherapy. 2017;103(1):1-12.

8. WHITE PD, MALLORY GK, SALCEDO-SALGAR J. The Speed of Healing of Myocardial Infarcts. Trans Am Clin Clim Assoc. 1936;52:97-105.

9. LAMERS HJ, DROST WS, KROON BJ, VAN ES LA, MEILINK-HOEDEMAKER LJ, BIRKENHÄGER WH. Early mobilization after myocardial infarction: a controlled study. Br Med J [Internet]. 1973 Feb 3 [cited 2016 Aug 20];1(5848):257-9. Available from: http://www.ncbi.nlm.nih. gov/pubmed/4685317.

10. LÓPEZ-JIMÉNEZ F, PÉREZ-TERZIC C, ZEBALLOS P, ANCHIQUE C, BURDIAT G, GONZÁLEZ K, et al. Consenso de Rehabilitación Cardiovascular y Prevención Secundaria de las Sociedades Interamericana y Sudamericana de Cardiología. Rev Urug Cardiol. 2013;28:189-224.

11. ANCHIQUE SANTOS CV, LOPEZ-JIMENEZ F, BENAIM B, BURDIAT G, FERNANDEZ CORONADO R, GONZALEZ $\mathrm{G}$, et al. Cardiac Rehabilitation in Latin America. Prog Cardiovasc Dis [Internet]. 2014 Nov [cited 2017 Jun 27];57(3):26875. Available from: http://linkinghub.elsevier.com/retrieve/pii/ S0033062014001388.

12. SANTIBÁÑEZ C. Situación actual de la rehabilitación cardiaca en Chile. Rev Med Chile [Internet]. 2012;140:561-8. Available from: http://www.scielo.cl/scielo.php?script=sci_arttext\&pid=S0034-98872012000500002

13. NYDAHL P, SRICHAROENCHAI T, CHANDRA S, KUNDT FS, HUANG M, FISCHILL M, et al. Safety of patient mobilization and rehabilitation in the intensive care unit: Systematic review with meta-analysis. Ann Am Thorac Soc.
2017;14(5):766-77.

14. HODGSON CL, STILLER K, NEEDHAM DM, TIPPING CJ, HARROLD M, BALDWIN CE, et al. Expert consensus and recommendations on safety criteria for active mobilization of mechanically ventilated critically ill adults. Crit Care. 2014;18(6):658.

15. FARDY PS, YANOWITZ FG. Rehabilitación Cardiaca. La forma física del adulto y las pruebas de esfuerzo. PAIDOTRIBO, editor. 2003. $414 \mathrm{p}$.

16. PESCATELLO L. ACSM's Guidelines for Exercise Testing and Prescripción. Ninth. 2014. 456 p.

17. AINSWORTH BE, HASKELL WL, WHITT MC, IRWIN ML, SWARTZ AM, STRATH SJ, et al. Compendium of Physical Activities: an update of activity codes and MET intensities. [cited 2017 Aug 4]; Available from: http://www.juststand.org/ portals/3/literature/compendium-of-physical-activities.pdf

18. BLANKSTEIN R, WARD RP, ARNSDORF M, JONES B, LOU Y-B, PINE M. Female gender is an independent predictor of operative mortality after coronary artery bypass graft surgery: contemporary analysis of 31 Midwestern hospitals. Circulation [Internet]. 2005 Aug 30 [cited 2018 Jun 10];112(9 Suppl):I323-7. Available from: http://www.ncbi.nlm.nih.gov/ pubmed/16159840.

19. LIN W-C, HO C-H, TUNG L-C, HO C-C, CHOU W, WANG C-H. Differences Between Women and Men in Phase I Cardiac Rehabilitation After Acute Myocardial Infarction A Nationwide Population-Based Analysis. Medicine (Baltimore) [Internet]. 2016 [cited 2017 Jun 27];95(3):e2494. Available from: https:// www.ncbi.nlm.nih.gov/pmc/articles/PMC4998261/pdf/medi-95-e2494.pdf.

20. OVEREND TJ, ANDERSON CM, JACKSON J, LUCY SD, PRENDERGAST M, SINCLAIR S. Physical therapy management for adult patients undergoing cardiac surgery: a canadian practice survey. Physiother Can [Internet]. 2010 [cited 2017 Dec 28];62(3):215-21. Available from: http://www.ncbi.nlm. nih.gov/pubmed/21629599 